\title{
ELEMENTARY PROOF OF A THEOREM ON LORENTZ MATRICES
}

\author{
E. R. VAN KAMPEN
}

Let $x$ and $y$ be real $n$ - and $m$-vectors and $x^{2}, y^{2}$ the scalar squares of $x, y$. The corresponding Lorentz matrices are matrices of $(n+m)$ dimensional real linear transformations which leave the quadratic form $x^{2}-y^{2}$ invariant. Let the transformation be written in the form

$$
\left(\begin{array}{ll}
A & B \\
C & D
\end{array}\right)\left(\begin{array}{l}
x \\
y
\end{array}\right)=\left(\begin{array}{l}
A x+B y \\
C x+D y
\end{array}\right) .
$$

Then the signs of the determinants $|A|$ and $|D|$ form two 1-dimensional representations of the Lorentz group. Two algebraic proofs at present available for this fact ${ }^{1}$ depend on a recursive factorization of the Lorentz matrix into simple factors or on deeper facts from the theory of representations. On the other hand, a simple topological proof may be given in quite an obvious manner. In this note the topological proof is briefly sketched and then a simple algebraic proof is given which does not depend on recursive factorization or representation theory and is valid in any real field.

The set defined by $x^{2}-y^{2} \geqq 1$ in the real $(n+m)$-dimensional space possesses one basic $(n-1)$-dimensional (finite) cycle $\Gamma$ which can most easily be represented by the $(n-1)$-dimensional basic cycle of the $(n-1)$-dimensional sphere $x^{2}=1, y=0$. Now $\Gamma$ is transformed by (1) into a cycle homologous to $+\Gamma$ or to $-\Gamma$ according as $|A|$ is positive or negative. The formal proofs of these topological facts are obtained most easily from the remark that the whole space $x^{2}-y^{2} \geqq 1$ can be retracted into its subset $x^{2}=1, y=0$ by a deformation which does not change the value of $x /\left(x^{2}\right)^{1 / 2}$ for any point. That sign $|A|$ is a one-dimensional representation of the Lorentz group is of course evident from the fact that $\Gamma$ is transformed by (1) into a cycle homologous to sign $|A| \cdot \Gamma$. The statement concerning the signature of $|D|$ depends on a similar consideration for the set defined by $x^{2}-y^{2} \leqq-1$.

Now let the elements of the matrix in (1) belong to any real field. Let the unit matrices of dimensions $n$ and $m$ be denoted by $E_{n}$ and $E_{m}$. The fact that the matrix in (1) is a Lorentz matrix may be expressed by the relations:

1 Cf. W. Givens, Factorization and signatures of Lorentz matrices, this Bulletin, vol. 46 (1940), pp. 81-85, where other references are given. My thanks are due to Dr. Murnaghan who drew my attention to the above theorem. 


$$
A^{\prime} A-C^{\prime} C=E_{n}, \quad D^{\prime} D-B^{\prime} B=E_{m}, \quad A^{\prime} B=C^{\prime} D,
$$

which may be obtained by forming the expression $x^{2}-y^{2}$ for the vector on the right in (1).

If $P$ is a matrix of $m$ rows and $n$ columns, such that $E_{n}-P^{\prime} P$ is positive definite, then the sign of the determinant $|A+B P|$ is independent of $P$; in particular $|A+B P| \neq 0$ and $|A| \neq 0$.

In fact, from (2) one easily obtains the identity

$$
(A+B P)^{\prime}(A+B P)=(C+D P)^{\prime}(C+D P)+E_{n}-P^{\prime} P .
$$

Since $E_{n}-P^{\prime} P$ is assumed to be positive definite, this implies that $(A+B P)^{\prime}(A+B P)$ is positive definite. Thus $|A+B P| \neq 0$ and, by choosing $P=0$, also $|A| \neq 0$. On replacing $P$ by $t P$, one sees that the determinant $|A+t B P|$, which is a polynomial in the parameter $t$, is never 0 while $-1 \leqq t \leqq 1$. For $E_{n}-t^{2} P^{\prime} P=E_{n}-P^{\prime} P+\left(1-t^{2}\right) P^{\prime} P$ is positive definite if $-1 \leqq t \leqq 1$. Thus the polynomial $|A+t B P|$ cannot change its sign as $t$ varies between 0 and 1 . In the field of real numbers this is evident. If the underlying field is any real field, and if the polynomial $|A+t B P|$ took both possible signs for $-1 \leqq t \leqq 1$, then one could adjoin to the field a root of $|A+t B P|=0$ which lies between -1 and 1 . In the enlarged field one obtains of course a contradiction with the fact that $|A+t B P| \neq 0$ for $-1 \leqq t \leqq 1$.

Let the product of two Lorentz matrices be written in the form

$$
\left(\begin{array}{ll}
A_{1} & B_{1} \\
C_{1} & D_{1}
\end{array}\right)\left(\begin{array}{ll}
A_{2} & B_{2} \\
C_{2} & D_{2}
\end{array}\right)=\left(\begin{array}{ll}
A_{1} A_{2}+B_{1} C_{2} & A_{1} B_{2}+B_{1} D_{2} \\
C_{1} A_{2}+D_{1} C_{2} & C_{1} B_{2}+D_{1} D_{2}
\end{array}\right) .
$$

Then one has

$$
A_{1} A_{2}+B_{1} C_{2}=\left(A_{1}+B_{1} C_{2} A_{2}^{-1}\right) A_{2}=\left(A_{1}+B_{1} P\right) A_{2},
$$

where $P=C_{2} A_{2}^{-1}$. But

$$
\begin{aligned}
E_{n}-P^{\prime} P & =E_{n}-\left(A_{2}^{\prime}\right)^{-1} C_{2}^{\prime} C_{2} A_{2}^{-1}=\left(A_{2}^{\prime}\right)^{-1}\left(A_{2}^{\prime} A_{2}-C_{2}^{\prime} C_{2}\right) A_{2}^{-1} \\
& =\left(A_{2}^{\prime}\right)^{-1} A_{2}^{-1}
\end{aligned}
$$

is a positive definite matrix, so that sign $\left|A_{1}+B_{1} C_{2} A_{2}^{-1}\right|=\operatorname{sign}$ $\left|A_{1}+B_{1} P\right|=\operatorname{sign}\left|A_{1}\right|$. Thus

$$
\operatorname{sign}\left|A_{1} A_{2}+B_{1} C_{2}\right|=\operatorname{sign}\left|A_{1}\right| \cdot \operatorname{sign}\left|A_{2}\right| \text {. }
$$

This completes the algebraic proof of the above theorem.

The geometrical content of the proof becomes clearer, if one realizes that the $n$-dimensional linear spaces with the equations $y=P x$ (where $E_{n}-P^{\prime} P$ is positive definite) are precisely those spaces through the origin which meet the quadratic $x^{2}-y^{2}=1$ in a completely elliptical 
quadratic (and the cone $x^{2}-y^{2}=0$ in its vertex only). Thus this system of linear spaces is invariant under the Lorentz group. That the sign of $|A+B P|$ is independent of $P$ means that the orientation of all spaces $y=P x$ is left invariant by a Lorentz matrix with $|A|>0$ and is changed into its opposite by a Lorentz matrix with $|A|<0$. Complications in preceding proofs of the theorem apparently originate either from the inclusion of the proof that every matrix $P$ with positive definite $E-P^{\prime} P$ is the matrix $C A^{-1}$ of a Lorentz transformation (1) and/or of the proof that the subgroup of the Lorentz group defined by $|A|>0,|D|>0$ is connected.

The 1-dimensional representation of the Lorentz group given by the determinants of the Lorentz matrix (1) is the product of the two representations given by the signs of $|A|$ and $|D|$. In fact, $D$ as well as $A$ is nonsingular. Thus ${ }^{2}$

$$
\begin{aligned}
\left|\begin{array}{ll}
A & B \\
C & D
\end{array}\right| & =|D| \cdot\left|\begin{array}{ll}
A & B \\
D^{-1} C & E_{m}
\end{array}\right|=|D| \cdot\left|\begin{array}{ll}
A-B D^{-1} C & 0 \\
D^{-1} C & E_{m}
\end{array}\right| \\
& =|D| \cdot\left|A-B D^{-1} C\right|,
\end{aligned}
$$

so that, since $B D^{-1}=A^{\prime-1} C^{\prime}$ and $\left|A^{\prime}\right|=|A|$,

$$
\left|\begin{array}{ll}
A & B \\
C & D
\end{array}\right|=|D| \cdot\left|A-A^{\prime-1} C^{\prime} C\right|=\frac{|D|}{|A|}\left|A^{\prime} A-C^{\prime} C\right|=\frac{|D|}{|A|} .
$$

Thus the sign of

$$
\left|\begin{array}{ll}
A & B \\
C & D
\end{array}\right|
$$

is the product of the signs of $|D|$ and $|A|$. Since

$$
\left|\begin{array}{ll}
A & B \\
C & D
\end{array}\right|=\frac{|A|}{|D|}
$$

may be similarly proved, one has

$$
\left|\begin{array}{ll}
A & B \\
C & D
\end{array}\right|=\operatorname{sign}|A| \cdot \operatorname{sign}|D| \text {. }
$$

The Johns Hopkins University

${ }^{2} \mathrm{Cf}$. J. Williamson, The expansion of determinants of composite order, American Mathematical Monthly, vol. 40 (1933), pp. 65-69. 